\title{
High CpG Island Methylator Phenotype
}

National Cancer Institute

\section{Source}

National Cancer Institute. High CpG Island Methylator Phenotype. NCI Thesaurus. Code C150486.

A phenotype characterized by hypermethylation of a high number of promoterassociated CpG islands. 